\title{
Diagnostic utility of probe-based confocal laser endomicroscopy in superficial non-ampullary duodenal epithelial tumors
}

\section{다 (1) $\odot$}

\author{
Authors \\ Tomomitsu Tahara ${ }^{1}$, Noriyuki Horiguchi ${ }^{1}$, Tsuyoshi Terada, Hyuga Yamada ${ }^{1}$, Dai Yoshida ${ }^{1}$, Masaaki Okubo ${ }^{1}, K^{\prime}$ Khei \\ Funasaka1, Mitsuo Nagasaka', Yoshihito Nakagawa', Tetsuya Tsukamoto², Tomoyuki Shibata', Makoto Kuroda², \\ Naoki Ohmiya ${ }^{1}$
}

Institutions

1 Department of Gastroenterology, Fujita Health University School of Medicine, Toyoake, Japan

2 Department of Diagnostic Pathology I, School of Medicine, Fujita Health University, Toyoake, Japan

submitted 10.4 .2019

accepted after revision 17.7.2019

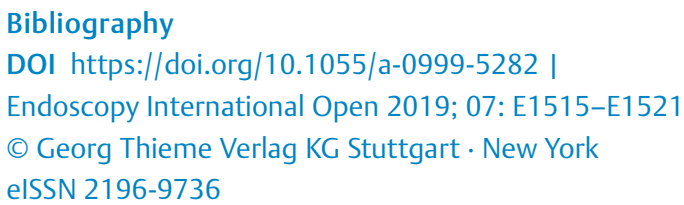

Corresponding author

Tomomitsu Tahara, 1-98 Dengakugakubo Kutsukake-cho, Toyoake, Aichi, 470-1192, Japan

Fax: +81-562-93-8300

tomomiccyu@yahoo.co.jp

\section{ABSTRACT}

Background and study aims Endoscopic diagnosis of superficial non-ampullary duodenal epithelial tumors (SNADETs) has not been established. Probe-based confocal laser endomicroscopy (pCLE: Cellvizio) provides real-time endomicroscopic analysis. We developed and validated a new pCLE classification of SNADET based on abnormal findings. Patients and methods pCLE scanning of 20 SNADET lesions including 16 adenomas and four carcinomas was retrospectively evaluated to explore abnormal pCLE findings in relation to histological features. Diagnostic yield of $\mathrm{pCLE}$ findings was prospectively evaluated in an additional 20 SNADET lesions including 16 adenomas and four carcinomas.

Results In a retrospective study, we identified four abnormal pCLE findings of SNADETs: (1) dark epithelium, (2) columnar cells irregularly extending to the lumen, (3) distorted crypt structure, and (4) fluorescein leakage. Dark epithelium distinguished neoplastic lesions (adenomas and carcinomas) from non-neoplastic duodenal mucosa with a sensitivity of $90 \%$ and a specificity of $100 \%$. Distorted crypt structure distinguished carcinomas from adenomas and non-neoplastic duodenal mucosa with a sensitivity of $100 \%$ and a specificity of $94 \%$. In the prospective study, the sensitivity and the specificity of the dark epithelium for the diagnosis of neoplastic lesions (adenomas + carcinomas) was $75 \%$ and $100 \%$. Sensitivity and the specificity of the distorted crypt structure for discrimination of carcinoma from adenoma were $100 \%$ and $94 \%$, respectively.

Conclusions The PCLE findings correlated with the histopathology of the SNADETs. Dark epithelium and distorted crypt structure were informative pCLE findings to predict presence of neoplasia and cancer in the SNADET, respectively.

\section{UMIN-CTR}

UMIN000013857

TRIAL REGISTRATION: Single-Center, prospective observational trial UMIN000013857 at upload.umin.ac.jp

\section{UMIN-CTR}

UMIN000033100

TRIAL REGISTRATION: Single-Center, prospective observational trial UMIN000033100 at upload.umin.ac.jp

\section{Introduction}

Superficial non-ampullary duodenal epithelial tumors (SNADETs) are neoplastic epithelial lesions, arising in the duodenal bulb and descending portion. It has been reported that widespread use of endoscopy has increased detection of SNADETs
$[1,2]$. SNADETs are considered to be precancerous or earlystage cancerous lesions and histological subtypes consist of adenoma and adenocarcinoma. Precise endoscopic diagnosis for predicting the histopathology of SNADETS is important in considering the appropriate treatment. 
Thus far, an endoscopic diagnostic algorithm for SNADETs has not been established. In addition, consistency of the histological diagnosis among pretreatment endoscopic biopsy and the resected specimen is low [3]. Most SNADETs are treated by endoscopic resection; however, biopsy induces severe fibrosis, which makes the endoscopic treatment difficult. An alternative endoscopic diagnostic strategy is therefore needed for precise prediction of the histopathology of SANDETs.

Probe-based confocal laser endomicroscopy ( $p C L E$ ) is an imaging technique used to provide in vivo histological information during endoscopy and enables real-time optical biopsy. These microscopic images are obtained by inserting a confocal miniprobe (Cellvizio, Mauna Kea Technologies, France) through the working channel of an endoscope. The principle of pCLE is based on tissue reflectance or tissue fluorescence after application of fluorescence agents, such as fluorescein sodium, generating images with 1000 -fold magnification ability, with a quality nearly equivalent to the pathological examination of biological samples $[4,5]$. The potential diagnostic utility of confocal imaging has been proposed in a variety of gastrointestinal diseases including neoplastic and inflammatory lesions, such as Barrett's esophagus [6,7], gastric carcinoma [8], colorectal polyps $[9,10]$, and inflammatory bowel diseases [11]. Various classification systems have been established to diagnose disease entities of the gastrointestinal tract [6-11], but reports showing the diagnostic utility for duodenal neoplastic lesions have been relatively rare $[12,13]$. A previous study from Japan reported a novel pCLE classification of SNADETs, generating two pCLE findings. One was the $A B C$ sign, defined as brightwhite cytoplasm with sharp gland structures, which is related to low-grade intraepithelial neoplasia (LGIEN). The other was the Chaos sign, defined as a blackish crypt with some white regions that are heterogeneously distributed and have a poorly defined gland structure. This finding is related to high-grade intraepithelial neoplasia (HGIEN) [13]. This simplified and intuitive classification suggested the potential utility of $\mathrm{pCLE}$ for di- agnosis of SNADETs. On the other hand, our preliminary experience suggests that even low-grade adenomas have a variety of abnormal pCLE findings within a tumor, possibly reflecting its histological features [14]. In this study, we explored a new pCLE classification of SNADETs based on their various abnormal findings. Frequency of abnormal pCLE findings was retrospectively investigated in SNADET lesions, including adenomas and carcinomas. We sought to validate our classification in a subsequent prospective study.

\section{Patients and methods}

\section{Patients}

We studied 40 SNATET lesions from 26 patients attending the endoscopy center of Fujita Health University between April 2015 and December 2017. The 40 lesions consisted of two cohorts of patients during two different periods: the first cohort (20 lesions from 16 patients between April 2015 and December 2016,) and the second cohort (20 lesions from 11 patients between January 2017 and December 2017). One patient overlapped the first and second cohorts; that patient was diagnosed with a new SNADET lesion in a follow-up endoscopic examination after initial endoscopic resection of a SNADET lesion. No patients included in this study had evidence of familial adenomatous polyposis (FAP). Clinicopathological features including age, gender, anatomical location, size, morphologic appearance according to the Paris classification are summarized in the $>$ Table 1. This study was reviewed and approved by the Institutional Review Board of Fujita Health University School of Medicine and was registered with the University Hospital Medical Information Network (UMIN000013857, UMIN000033100). Written informed consent was obtained from all participating subjects.

- Table 1 Characteristics of study patients

\begin{tabular}{|l|l|l|}
\hline Variables & $\mathbf{1 s t}$ cohort & 2nd cohort \\
\hline Patient number & 16 & 11 \\
\hline Period & April 2015-December 2017 & January 2017-December 2017 \\
\hline Lesion number & 20 & 20 \\
\hline Median age (range) & $68(42-85)$ & $72(45-83)$ \\
\hline Male/Female & $12 / 4$ & $8 / 3$ \\
\hline Location: duodenal bulb/second part & $3 / 17$ & $1 / 19$ \\
\hline Macroscopic type (according to the Paris classification': & $4 / 11 / 5$ & $2 / 12 / 4 / 2$ \\
\hline $0-$ I/0-Ila/0-Ila + c/0-IIc) & & $11.9 \pm 10.1$ \\
\hline Tumor size (mean \pm SD, mm) & $14.2 \pm 10.5$ & $16 / 4$ \\
\hline Histological type: adenoma/carcinoma & $16 / 4$ & $20 / 0$ \\
\hline Treatment: endoscopic resection/pancreatoduodenectomy & $18 / 2$ & \\
\hline 1 0-I/0-Ila/0-Ila +c/0-Ilc correspond to polypoid/flat elevated/flat elevated plus depressed lesions.
\end{tabular}




\section{Endoscopic procedure}

All endoscopic procedures were performed by conventional white-light observation followed by PCLE. All procedures were under conscious sedation using either intravenous (IV) midazolam (4-6mg) or diazepam (5-10 mg). For conventional whitelight observation, the Olympus GIF-H260Z and CV260SL/ CV290SL (Olympus Medical Systems, Tokyo, Japan) were used. The pCLE was performed using a high-definition miniprobe (Cellvizio), with a field of view of $240 \mu \mathrm{m}$, a lateral resolution of $1 \mu \mathrm{m}$ and confocal depth of approximately $60 \mu \mathrm{m}$. Initially, endoscopic pictures for each lesion were taken in the order of conventional white-light. Subsequently, pCLE was performed by inserting the pCLE probe into the working channel of the endoscope. Before pCLE scanning, fluorescein sodium $(2.5 \mathrm{~mL}$, $10 \%$ ) was injected IV for tissue contrast. Thirty to sixty seconds after fluorescein sodium injection, the tip of the miniprobe was placed on the background normal mucosa to obtain a control image, and pCLE scanning was performed on the targeted lesions as well as their adjacent normal appearing duodenal mucosa. Targeted lesions and adjacent normal-appearing duodenal mucosa were then subjected to PCLE scanning. The PCLE scanning of targeted lesions and the adjacent mucosa was recorded in a video of 1 minute or longer. All endoscopic procedures, including the white-light observation and the PCLE, were performed by three experienced endoscopists (T.T, N.H. and N.O.).

\section{Retrospective study}

For the first cohort of 20 SNADET lesions (16 adenomas and 4 carcinomas), pCLE scanning of the targeted lesions and the adjacent normal mucosa was retrospectively evaluated and suggestive abnormal pCLE findings were examined in relation to their histological findings. To define suggestive pCLE findings to distinguish non-neoplastic and neoplastic tissue in SNADET lesions, we consulted several reports about pCLE findings of neoplastic tissues in the gastrointestinal tract [4, 6-8, 15-17]. Features capable of distinguishing gastrointestinal non-neoplastic and neoplastic tissue by pCLE imaging have been summarized in the Miami Classification system [4]. In general, nonneoplastic epithelium is characterized by well-organized brightly colored uniform crypts, while neoplastic tissue such as adenoma and carcinoma appears as dark-colored epithelium. Irregularity and heterogeneity of individual crypts would be also be associated with the atypical degree of neoplastic tissue [4]. Based on this issue, abnormal findings of PCLE in the duodenal lesion were defined by presence of (1) dark epithelium, (2) columnar cells irregularly extending to the lumen, and (3) distorted crypt structure. We also investigated presence of (4) fluorescein leakage, which is thought to be a PCLE finding of tissue vulnerability in inflammatory bowel diseases (\$ Fig. 1) [11]. This finding may also be associated with neoplastic cells with the loss of cell-cell adhesion [17]. Presence of these four abnormal findings were determined in a >1-minute confocal video from 20 SNADET lesions and the adjacent normal mucosa. This assessment was based on the consensus of two observers (T.T. and N.H.), who had previously performed more than 100 pCLE examinations of gastrointestinal diseases, including neoplastic and inflammatory lesions. They had previously reviewed the pathological findings and pCLE findings from the above cases to learn about suggestive PCLE findings of gastrointestinal diseases related to the pathological findings. Before the current study, both observers reviewed the medical literature $[4,6-8$, $15-17$ ] to understand the abnormal pCLE findings described in this study. The pCLE scanning of all lesions was always evaluated by both observers together to assess presence of abnormal pCLE findings.

\section{Prospective study}

For the second cohort from 20 SNADET lesions (16 adenomas and 4 carcinomas), diagnostic yield of the two pCLE findings was prospectively evaluated, and the final pathological diagnosis was not available at enrollment. All pCLE image evaluation was performed in real -time during the PCLE examination based on the consensus of two observers (T.T. and N.H.). The diagnosis was immediately recorded and was never changed once completed.

\section{Statistical analysis}

Categorical variables were determined using the Chi-Squared test. The diagnostic utility of pCLE findings for predicting the pathology of SNADETs was expressed as sensitivity and specificity, positive predictive value (PPV) and negative predictive value (PPV). A $95 \%$ confidence interval $(95 \% \mathrm{Cl})$ was also calculated. $P<0.05$ was considered to be statistically significant.

\section{Results \\ Retrospective study}

Frequency of the four abnormal pCLE findings in the 20 SNADET lesions in the retrospective cohort is shown in $>$ Table 2 . There was no disagreement in assessment of pCLE findings among all 20 SNADET lesions and the normal adjacent mucosa between the two observers (T.T. and N.H.). Normal duodenal mucosa did not exhibit any abnormal findings, while all four carcinomas had abnormal findings with a frequency of $100 \%$. On the other hand, the 16 adenomas had the findings with various percentages. Frequency of all four of the pCLE findings was significantly associated with histopathology of SNADETS by the Chi-Squared test using the $2 \times 3$ contingency table (all $P<0.0001$ ). In particular, dark epithelium distinguished neoplastic lesions (adenomas + carcinomas) from non-neoplastic mucosa with a sensitivity of $90 \%(95 \% \mathrm{Cl}, 0.79-0.90)$, a specificity of $100 \%(95 \% \mathrm{Cl}$, $0.89-1.00)$, a PPV of $100 \%(95 \% \mathrm{Cl}, 0.88-1.00)$ and a NPV of $91 \%(95 \% \mathrm{Cl}, 0.81-0.91)$. Distorted crypt structure distinguished carcinomas from adenomas with a sensitivity of $100 \%$ (95\% Cl, $0.60-1.00)$, specificity of $94 \%(95 \% \mathrm{Cl}, 0.85-0.94)$, PPV of $80 \%(95 \% \mathrm{Cl}, 0.48-0.80)$ and NPV of $100 \%(95 \% \mathrm{Cl}$, $0.90-1.00)$. $>$ Fig. 2 shows representative pCLE findings of normal mucosa, adenoma and cancer with their histological findings. Normal mucosa was characterized as uniform and brightcolored villi structures. Adenomas showed small and dark-colored crypt structures, and carcinomas showed irregular- 

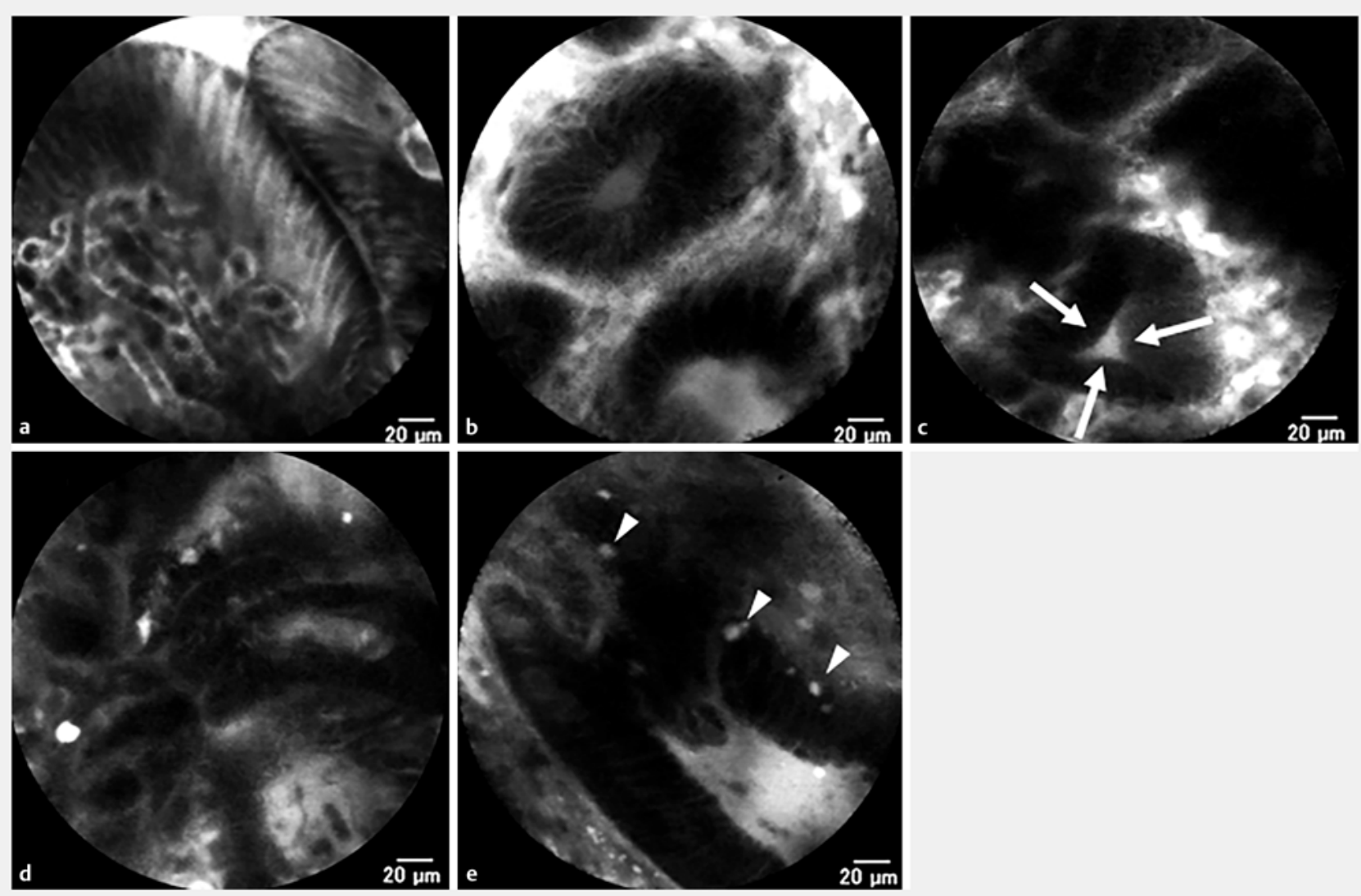

- Fig. 1 Representative PCLE findings of the normal duodenal mucosa and the SNADETs. a Normal duodenal mucosa. b Dark epithelium. c Columnar cells irregularly extending to the lumen (white arrows). d Distorted crypt structure. e Fluorescein leakage (white arrowheads).

- Table 2 Frequency of abnormal PCLE findings of SNADETs and adjacent normal mucosa in the retrospective study.

\begin{tabular}{|l|l|l|l|}
\hline Variables & Adjacent normal mucosa & Adenoma & Carcinoma \\
\hline & $(n=20)$ & $(n=16)$ & $(n=4)$ \\
\hline Dark epithelium $^{1}$ & $0 \%(0 / 20)$ & $87.5 \%(14 / 16)$ & $100.0 \%(4 / 4)$ \\
\hline Columnar cells irregularly extending to the lumen & $0 \%(0 / 20)$ & $56.3 \%(9 / 16)$ & $100.0 \%(4 / 4)$ \\
\hline Distorted crypt structure $^{2}$ & $0 \%(0 / 20)$ & $6.3 \%(1 / 16)$ & $100.0 \%(4 / 4)$ \\
\hline Fluorescein leakage & $0 \%(0 / 20)$ & $56.3 \%(9 / 16)$ & $100.0 \%(4 / 4)$ \\
\hline
\end{tabular}

$\mathrm{Cl}$, confidence interval; PPV, positive predictive value; NPV, negative predictive value.

${ }^{1}$ Diagnostic yield for predicting neoplastic lesions (adenoma + carcinoma): Sensitivity $90 \%$ with $95 \%$ Cl: $0.79-0.90$, Specificity $100 \%$ with $95 \%$ Cl: $0.89-1.00$, PPV $100 \%$ with $95 \% \mathrm{Cl}: 0.88-1.00$, NPV $91 \%$ with $95 \% \mathrm{Cl}: 0.81-0.91$.

${ }^{2}$ Diagnostic yield for discriminating carcinoma from adenomas: Sensitivity $100 \%$ with $95 \% \mathrm{Cl}: 0.60-1.00$, Specificity $94 \%$ with $95 \%$ Cl: $0.85-0.94$, PPV $80 \%$ with $95 \% \mathrm{Cl}: 0.48-0.80$, NPV $100 \%$ with $95 \% \mathrm{Cl}: 0.90-1.00$.

shaped crypt structures. All these findings were closely associated with histological findings.

\section{Prospective study}

Because the two abnormal findings, the dark epithelium and the distorted crypt structures, seemed to be useful, the diagnostic yield of these two pCLE findings was prospectively evaluated in an additional 20 SNADETs, including 16 adenomas and 4 cancers.
The result demonstrated that dark epithelium distinguished neoplastic lesions (adenomas + carcinomas) from non-neoplastic mucosa with a sensitivity of $75 \%(95 \% \mathrm{Cl}, 0.63-0.75)$, a specificity of $100 \%(95 \% \mathrm{Cl}, 0.88-1.00)$, a PPV of $100 \%(95 \% \mathrm{Cl}$, $0.84-1.00)$ and a NPV of $91 \%(95 \% \mathrm{Cl}, 0.71-0.80)$. The distorted crypt structure distinguished carcinomas from adenomas with a sensitivity of $100 \%(95 \% \mathrm{Cl}, 0.60-1.00)$, a specificity of $94 \%(95 \% \mathrm{Cl}, 0.85-0.94)$, a PPV of $80 \%(95 \% \mathrm{Cl}, 0.48-0.80)$ and a of NPV $100 \%(95 \% \mathrm{Cl}, 0.90-1.00)(\triangleright$ Table 3$)$. 


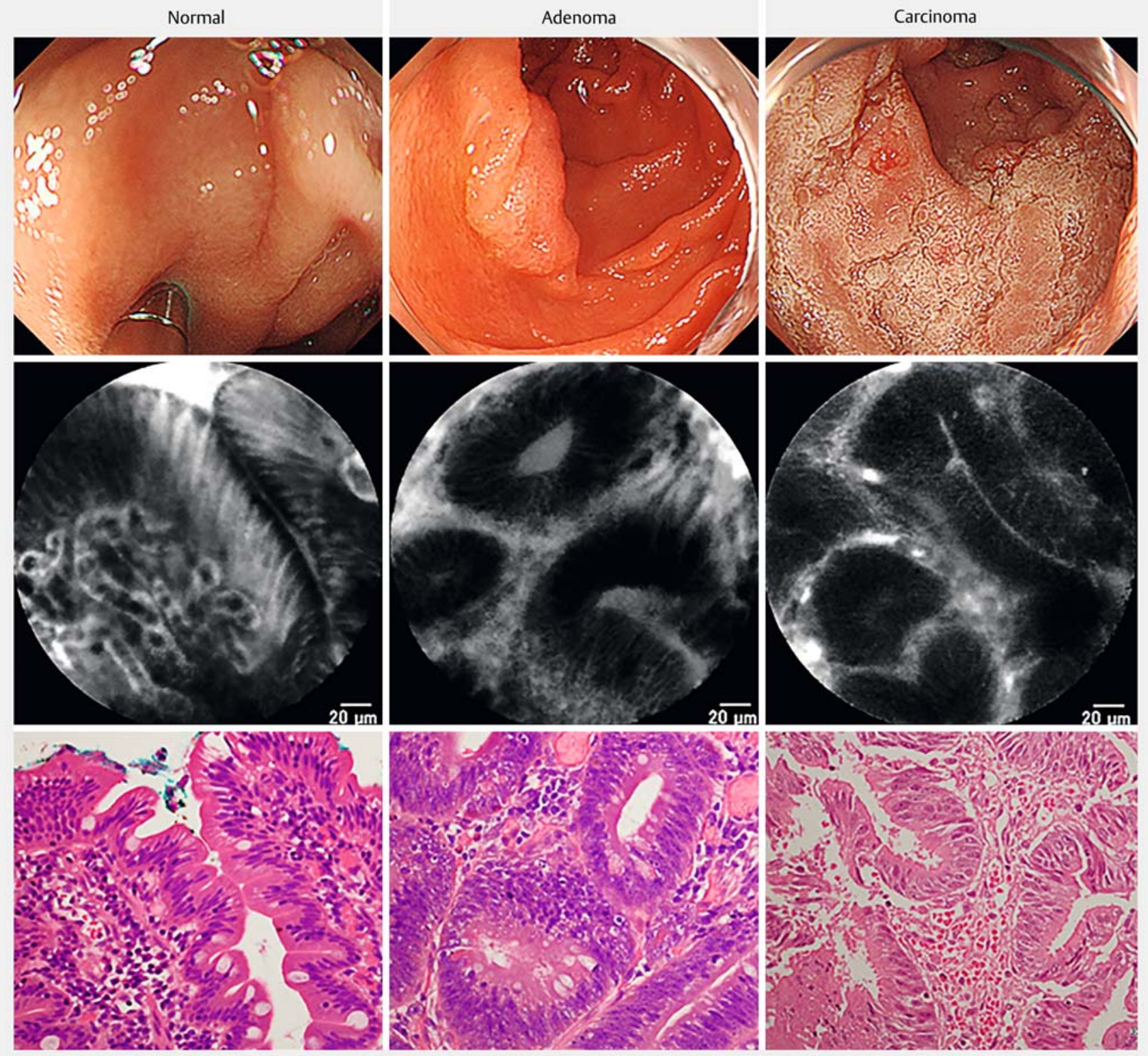

- Fig. 2 Conventional white light imaging endoscopy (upper), pCLE imaging (middle) and histopathology with hematoxylin-eosin staining (lower) of the normal duodenal mucosa (left), adenoma (middle) and carcinoma (right). The pCLE imaging of the normal duodenal mucosa is characterized by bright and uniform crypts (middle left), while the pCLE imaging of adenoma is characterized by uniformly arranged, but blackish colored, crypts (middle center). The pCLE imaging of carcinoma is characterized by blackish and distorted crypt structures (middle right).

- Table 3 Diagnostic yield of dark epithelium and distorted crypt structure in the prospective study.

\begin{tabular}{|l|l|l|l|}
\hline Variables & $\begin{array}{l}\text { Normal mucosa } \\
(\mathbf{n = 2 0 )}\end{array}$ & $\begin{array}{l}\text { Adenoma } \\
(\mathbf{n = 1 6 )}\end{array}$ & $\begin{array}{l}\text { Carcinoma } \\
(\mathbf{n = 4})\end{array}$ \\
\hline Dark epithelium $^{1}$ & $0 \%(0 / 20)$ & $68.8 \%(11 / 16)$ & $100 \%(4 / 4)$ \\
\hline Distorted crypt structure $^{2}$ & $0 \%(0 / 20)$ & $6.3 \%(1 / 16)$ & $100 \%(4 / 4)$ \\
\hline
\end{tabular}

$\mathrm{Cl}$, confidence interval; PPV, positive predictive value; NPV, negative predictive value.

${ }^{1}$ Diagnostic yield for predicting neoplastic lesions (adenoma + carcinoma): Sensitivity $75 \%$ with $95 \%$ Cl: $0.63-0.75$, Specificity $100 \%$ with $95 \%$ Cl: $0.88-1.00$,PPV $100 \%$ with $95 \% \mathrm{Cl}: 0.84-1.00, \mathrm{NPV} 80 \%$ with $95 \% \mathrm{Cl}: 0.71-0.80$.

2 Diagnostic yield for discriminating carcinoma from adenomas: Sensitivity $100 \%$ with $95 \% \mathrm{Cl}: 0.60-1.00$,Specificity $94 \%$ with $95 \% \mathrm{Cl}$ : $0.85-0.94$, PPV $80 \%$ with $95 \% \mathrm{Cl}: 0.48-0.80$, NPV $100 \%$ with $95 \% \mathrm{Cl}: 0.9-1.00$. 


\section{Adverse events}

There were no complications associated with pCLE or IV injection of fluorescein, including bleeding, perforation, aspiration pneumonia, and anaphylaxis.

\section{Discussion}

We defined four suggestive abnormal pCLE findings of SNADETs: (1) dark epithelium, (2) columnar cells irregularly extending to the lumen, (3) distorted crypt structure, and (4) fluorescein leakage. Prevalence of these findings correlated well with histologic changes from the normal mucosa to adenoma and carcinoma. The pCLE findings of adenoma and carcinoma in the gastrointestinal tract have been characterized as having dark-colored crypts $[4,15]$. The two findings, columnar cells irregularly extending to the lumen and the distorted crypt structure, have also been defined as PCLE findings of the neoplastic crypts $[4,16]$. Fluorescein leakage has been associated with inflammatory bowel disease [11], reflecting their tissue vulnerability, and may also be associated with neoplastic cells with the loss of cell-cell adhesion $[17,18]$. Our results suggest that these abnormal pCLE findings can also be applied to SNADET lesions to predict their histopathology. Among the four pCLE findings, dark epithelium and distorted crypt structure were confirmed to be informative pCLE findings to predict neoplasia and cancer in the SNADETs, respectively. In particular, sensitivity and NPV for the dark epithelium and specificity and PPV for the distorted crypt structure seemed to be favorable under clinical conditions. Studies have reported that larger tumor size, surface redness, and presence of concavity using conventional endoscopy are informative appearances to differentiate high-grade intraepithelial neoplasia from low-grade intraepithelial neoplasia in gastrointestinal epithelial lesions [19, 20]. Another study proposed that high-resolution magnifying endoscopy with narrow band imaging (ME-NBI) potentially predicts histopathology of SNADETs [21]. However, the diagnostic value of the above studies was not sufficiently accurate [19, 20] nor has it been prospectively validated [21]. The pCLE details microarchitecture of lesions with 1000-fold magnification ability, which would be more directly linked to their histopathological features compared to images obtained by conventional endoscopy and ME-NBI. Our results indicate that real-time in vivo histological information with pCLE scanning enables us to determine the precise endoscopic diagnosis of SNADETs, reflecting their histopathological features.

Concerning the association between pCLE findings and histological features, it is possible that both morphologic and mechanistic differences may reflect pCLE findings of SNADTETs. Indeed, irregularity and heterogeneity of individual crypts, seen by $P C L E$, is associated with the atypical degree of neoplastic tissue [4]. It is reasonable to expect that such histological features can be highlighted as the "distorted crypt structure," seen in carcinomas. On the other hand, neoplastic tissues also have mechanistic differences including differences in expression of cell-cell adhesion proteins and transporter proteins that modulate absorption and metabolism of pharmacological agents
[18]. Such differences may influence incorporation of fluorescein and may contribute to the "dark epithelium" appearance of neoplastic lesions in the SNADETs. Dark/irregular epithelium has also been reported to be a predictive PCLE finding to diagnose duodenal adenoma lesions seen in patients with familial adenomatous polyposis (FAP), which is associated with an increased risk of developing periampullary and non-ampullary adenoma [22]. These findings suggest that dark epithelium would be a useful endoscopic finding to diagnose neoplastic lesions in the duodenum.

Regarding the other reports on CLE in duodenal lesions, Shahid et al. reported usefulness of discriminating dysplastic lesions from duodenal polyps using CLE by applying diagnostic criteria of dysplasia of Barrett's esophagus; however, the study lacked a diagnostic criterion discriminating adenoma and carcinoma [12]. Pittayanon et al. reported usefulness of real-time diagnosis using pCLE for duodenal adenoma lesions seen in patients with FAP [22], but the study did not examine cancerous lesions. Nonaka et al. developed pCLE classification to discriminate duodenal HGIEN lesions from LGIEN lesions [13]. The latter authors also reported a novel method to directly apply the fluorescein sodium on the duodenal lesion to provide confocal imaging. However, it should be noted that PCLE image acquisition can be avoided in the presence of thick mucosal mucous, which is often present in the gastrointestinal mucosa. In our study, reproducible clear images could be obtained within 1 minute after injection of fluorescein in all our cases. Such images can be interpreted for as long as 60 minutes [23]. Although possible adverse events associated with pCLE include allergic reactions to IV fluorescein, no complications associated with the IV injection of fluorescein were observed in our study cohort, suggesting that PCLE with the injection is feasible and safe.

\section{Conclusion}

In summary, pCLE findings correlated well with histopathology of the SNADETs. Dark epithelium and distorted crypt structure were informative $\mathrm{PCLE}$ findings to predict neoplasia and cancer in the SNADETs, respectively. Our results, especially the carcinoma cases, were derived from small cohorts of cases that need to be confirmed in a larger multicenter study. Our results also need to be evaluated in a study involving both neoplastic and non-neoplastic epithelial duodenal tumors to establish a diagnostic algorithm for PCLE in SNADETs.

\section{Competing interests}

None 


\section{References}

[1] Seifert E, Schulte F, Stolte M. Adenoma and carcinoma of the duodenum and papilla of Vater: a clinicopathologic study. Am J Gastroenterol 1992; 87: $37-42$

[2] Okada K, Fujisaki J, Kasuga A et al. Sporadic nonampullary duodenal adenoma in the natural history of duodenal cancer: a study of followup surveillance. Am J Gastroenterol 2011; 106: 357-64

[3] Kinoshita S, Nishizawa T, Ochiai Y et al. Accuracy of biopsy for the preoperative diagnosis of superficial nonampullary duodenal adenocarcinoma. Gastrointest Endosc 2017; 86: 329-32

[4] Wallace M, Lauwers GY, Chen Y et al. Miami classification for probebased confocal laser endomicroscopy. Endoscopy 2011; 43: 882 - 91

[5] Neumann H, Kiesslich R, Wallace MB et al. Confocal laser endomicroscopy: technical advances and clinical applications. Gastroenterology 2010; 139: 388-92.e1-2

[6] Canto MI, Anandasabapathy S, Brugge W et al. Confocal Endomicroscopy for Barrett's Esophagus or Confocal Endomicroscopy for Barrett's Esophagus (CEBE) Trial Group. In vivo endomicroscopy improves detection of Barrett's esophagus-related neoplasia: a multicenter international randomized controlled trial (with video). Gastrointest Endosc 2014; 79: $211-221$

[7] Kiesslich R, Gossner L, Goetz M et al. In vivo histology of Barrett's esophagus and associated neoplasia by confocal laser endomicroscopy. Clin Gastroenterol Hepatol 2006; 4: 979-987

[8] Li Z, Yu T, Zuo XL et al. Confocal laser endomicroscopy for in vivo diagnosis of gastric intraepithelial neoplasia: a feasibility study. Gastrointest Endosc 2010; 72: 1146 - 1153

[9] Buchner AM, Shahid MW, Heckman MG et al. Comparison of probebased confocal laser endomicroscopy with virtual chromoendoscopy for classification of colon polyps. Gastroenterology 2010; 138: 834 842

[10] Wanders LK, East JE, Uitentuis SE et al. Diagnostic performance of narrowed spectrum endoscopy, autofluorescence imaging, and confocal laser endomicroscopy for optical diagnosis of colonic polyps: a meta-analysis. Lancet Oncol 2013; 14: 1337-1347

[11] Lim LG, Neumann J, Hansen T et al. Confocal endomicroscopy identifies loss of local barrier function in the duodenum of patients with Crohn's disease and ulcerative colitis. Inflamm Bowel Dis 2014; 20: $892-900$

[12] Shahid MW, Buchner A, Gomez V et al. Diagnostic accuracy of probebased confocal laser endomicroscopy and narrow band imaging in detection of dysplasia in duodenal polyps. J Clin Gastroenterol 2012; 46: $382-389$

[13] Nonaka K, Ohata K, Ichihara S et al. Development of a new classification for in vivo diagnosis of duodenal epithelial tumors with confocal laser endomicroscopy: A pilot study. Dig Endosc 2016; 28: 186-193

[14] Tahara T, Horiguchi N, Nagasaka M et al. Probe-based confocal laser endomicroscopy ( $\mathrm{pCLE}$ ) images of submucosal growth of a duodenal mucous neck cell adenoma. Endoscopy 2016; 48: (Suppl. 01): E19E21

[15] Horiguchi N, Tahara T, Yamada H et al. In vivo diagnosis of early-stage gastric cancer found after Helicobacter pylori eradication using probebased confocal laser endomicroscopy. Dig Endosc 2018; 30: 219-227

[16] Ohmiya N, Horiguchi N, Tahara T et al. Usefulness of confocal laser endomicroscopy to diagnose ulcerative colitis-associated neoplasia. Dig Endosc 2017; 29: 626-633

[17] Royer C, Lu X. Epithelial cell polarity: a major gatekeeper against cancer? Cell Death Differ 2011; 18: 1470 - 1477

[18] Prueksapanich P, Pittayanon R, Rerknimitr R et al. Value of probebased confocal laser endomicroscopy ( $p C L E)$ and dual focus narrowband imaging $(\mathrm{dNBI})$ in diagnosing early squamous cell neoplasms in esophageal Lugol's voiding lesions. Endosc Int Open 2015; 3: E281 E288

[19] Oya M, Yao T, Nakamura T et al. Intestinal phenotypic expression of gastric depressed adenomas and the surrounding mucosa. Gastric Cancer 2003; 6: 179-84

[20] Kasuga A, Yamamoto Y, Fujisaki J et al. Clinical characterization of gastric lesions initially diagnosed as low-grade adenomas on forceps biopsy. Dig Endosc 2012; 24: 331 - 338

[21] Kikuchi D, Hoteya S, lizuka T et al. Diagnostic algorithm of magnifying endoscopy with narrow band imaging for superficial non-ampullary duodenal epithelial tumors. Dig Endosc 2014; 26: (Suppl. 02): $16-22$

[22] Pittayanon R, Rerknimitr R, Imraporn B et al. Diagnostic values of dual focus narrow band imaging and probe-based confocal laser endomicroscopy in FAP-related duodenal adenoma. Endosc Int Open 2015; 3 : E450-E455

[23] Becker V, von Delius S, Bajbouj M et al. Intravenous application of fluorescein for confocal laser scanning microscopy: evaluation of contrast dynamics and image quality with increasing injection-toimaging time. Gastrointest Endosc 2008; 68: 319-323 